\title{
Design and Implementation of a JXTA-Overlay P2P System and Smart Box Environment
}

\author{
Keita Matsuo $\nmid$, Leonard Barolli $\nmid$, Fatos Xhafa $\nmid \dagger$, \\ Akio Koyama $^{\dagger \ddagger}$, Arjan Durresi ${ }^{\ddagger \dagger}$, Makoto Takizawa ${ }^{\ddagger \ddagger}$ \\ † Graduate School of Engineering, Fukuoka Institute of Technology (FIT) \\ 3-30-1 Wajiro-Higashi, Higashi-Ku, 811-0295 Fukuoka, Japan \\ E-mail: bd07002@bene.fit.ac.jp \\ $\ddagger$ Department of Information and Communication Engineering \\ Fukuoka Institute of Technology (FIT) \\ 3-30-1 Wajiro-Higashi, Higashi-Ku, 811-0295 Fukuoka, Japan \\ E-mail: barolli@fit.ac.jp \\ †† Department of Languages and Informatics Systems, Polytechnic University of Catalonia \\ Jordi Girona 1-3, 08034 Barcelona, Spain \\ E-mail: fatos@1si.upc.edu \\ $\dagger \ddagger$ Department of Informatics, Yamagata University \\ 4-3-16 Jonan, Yonezawa 992-8510, Yamagata, Japan \\ E-mail: akoyama@eie.yz.yamagata-u.ac.jp \\ $\ddagger_{\dagger}$ Department of Computer and Information Science \\ Indiana University Perdue University Indianapolis \\ 723 W. Michigan Street SL 280, Indianapolis, IN 46202, USA \\ E-mail: durresi@cs.iupui.edu \\ 拉 Department of Computers and System Engineering, Tokyo Denki University \\ Ishizaka, Hatoyama, Saitama 350-0394, Japan \\ E-mail: taki@takilab.k.dendai.ac.jp
}

\begin{abstract}
In this paper, we present the design and implementation of the JXTA-based e-Learning P2P system. We also show the design and implementation of a smart box environment that will be used for stimulating the learners to increase the learning efficiency. The smart box is intended to be integrated with our P2P system as a useful tool for monitoring and controlling learners' activity. Finally, it is also useful to evaluate the feasibility of our P2P platform to integrate different computational devices.
\end{abstract}

\section{Introduction}

In the past few years we have observed an explosive growth of multimedia computing, communication and applications. Information Technology (IT) has a great effect in our everyday life and is transforming the way people live, work, and interact with each other, and is impacting the way businesses, government services, education, entertainment, and health care are operating.

The Internet is growing every day and the performance of computers is increased exponentially. However, the Internet architecture is based on Client/Server (C/S) topology, therefore can not use efficiently the clients features. It should be noted that computers of today have the same characteristics with conventional super-computers. Also, with appearance of new technologies such as ad-hoc networks, sensor networks, body networks, home networking, new network devices and application will appear. Therefore, it is very important to monitor, control and optimize these network devices via communication channels. However, in largescale networks such as Internet, it is very difficult to control the network devices, because of the security problems. Each network has its own security policy and the information should overcome the firewalls.

The firewalls are used for checking the signal information between private and public networks. The signals are transmitted according to some decided rules and it is very difficult to change the network security policy. So, there are many small networks and Intranets that do not allow the information coming from other networks. This happens especially in the networks of schools or libraries. Therefore, recently many researchers are working on Peer-to-Peer 
(P2P) networks, which are able to overcome the firewalls, NATs and other security devices without changing the network policy. The $\mathrm{P} 2 \mathrm{P}$ architecture is thus very important for future e-learning systems.

Virtual campuses and virtual universities are new ways of organizing students activities by using IT technologies. The virtual university is one of the most widely used form of virtual organizations in the today's teaching and learning activities. However, most of the current virtual organizations use server-mediated Internet-based applications that enable sharing among the members of the community.

In $\mathrm{C} / \mathrm{S}$ approaches the shared resources are centralized on servers and members of the virtual community (students, teachers, researchers, etc.) access them through light Web clients. Thus, every thing is done at server side while at the client side just an interface is needed. In general, the server-mediated approaches show several drawbacks, especially lack of scalability and efficiency in virtual campuses of many thousands of students.

The most powerful alternative to server-based approaches for applications of virtual universities is the use of decentralized approaches such as $\mathrm{P} 2 \mathrm{P}$ systems. In such systems, the computational burden of the system can be distributed to peer nodes of the system. Therefore, in decentralized systems users become themselves actors by sharing, contributing and controlling the resources of the systems. This characteristic makes $\mathrm{P} 2 \mathrm{P}$ systems very interesting for the development of decentralized applications [1,2] for virtual campuses and virtual organizations.

During the last decade, many e-learning systems are proposed and implemented, mostly using web technologies. With emerging of new technologies such as P2P, sensor and ad-hoc networks, it will be possible to build ubiquitous systems that can offer an equal educational environment regardless the region, age, time or the place [3,4].

This work is motivated by the need to develop decentralized P2P approaches to support learning and teaching activity in virtual universities. In this work, we present the design and implementation of main features of a JXTA-based P2P system (called JXTA-Overlay) that support in many ways the development of e-learning applications. JXTA-Overlay is thus a platform for developing group-ware applications. We also show the implementation and the design of a smart box environment that will be used for stimulating the learners to increase the learning efficiency. The smart box is integrated with the JXTA-Overlay by using efficient message sending between peers in the system.

The structure of this paper is as follows. In Section 2, we introduce the related work. In Section 3, we introduce the Project JXTA and explain JXTA-Overlay. In Section 4, we present the proposed P2P e-learning system. Finally, conclusions and future work are given in Section 5.

\section{Related Work}

In this section, we discuss the related work for P2P systems and Web based e-learning systems.

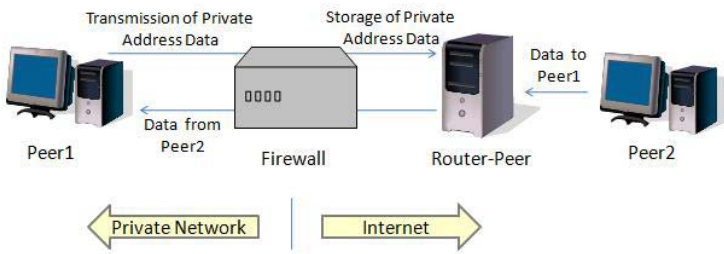

Figure 1. P2P communication.

Much of the initial research efforts and projects on $\mathrm{P} 2 \mathrm{P}$ systems were concerned with the investigation of overlay networks. It should be noted that each time more, this effort is oriented towards the design and implementation of libraries and platforms to support the development of real P2P applications, which could actually combine both Grid and $\mathrm{P} 2 \mathrm{P}$ technologies. Bal et al. [5] motivated the need for research from conceptual, algorithmic and application level tools to facilitate the application development task, that is, tools that enable writing, deploying and running Grid/P2P applications.

Since in $\mathrm{P} 2 \mathrm{P}$ applications file sharing plays an important role, a lot of research effort is being devoted to the development of reliable file sharing systems to be used in P2P applications [6]. In particular, several studies are done for measuring the efficiency in $\mathrm{P} 2 \mathrm{P}$ systems $[7,8,9]$ as it is crucial for such systems.

Due to the opportunities provided by the Internet, more and more people are taking advantage of online distance learning courses. During the last few years enormous research efforts have been dedicated to the development of elearning systems. Consequently, many large projects have been established [10, 11, 12, 13, 14, 15]. However, in these systems the e-learning completion rate is low. One of the reasons is the low study interest when the learner studies the subjects. Therefore, it is very important to stimulate learner's motivation during the study. In our previous works $[16,17]$, we proposed an agent based distance learning system to deliver appropriate studying materials to learners and stimulate learner's motivation.

However, all these systems are based on C/S architecture. In such approach the shared resources are centralized on servers and members of the virtual community (clients) access them through request protocols. Every thing is done at server side while at the client side just an interface is needed. Among the most important limitations of server-mediated approaches, we could distinguish lack of scalability, lack of fault tolerance, usually low performance/bottlenecks as well as high costs in acquiring, developing and maintaining such applications.

\section{JXTA Technology and JXTA-Overlay}

\subsection{JXTA technology}

JXTA technology [18] is a generalized group of protocols that allow different devices to communicate and collaborate among them. JXTA offers a platform covering basic needs in developing $\mathrm{P} 2 \mathrm{P}$ networks. 
By using JXTA framework is possible that a peer in a private network can be connected to a peer in the Internet by overcoming existing Firewalls as shown in Fig. 1. The procedure to overcome the fire-wall is as follows.

- In the Router Peer is stored the private address of Peer 1 by using the HTTP protocol to pass the firewall from Peer1.

- The Router Peer receives the data from Peer2 and access the Private address of Peer1 to transmit the data.

JXTA is an interesting alternative for developing P2P systems and groupware tools to support online teams of students in virtual campuses. In particular, it is appropriate for file sharing given that its protocols allow to develop either pure or mixed P2P networks. This last property is certainly important since pure $\mathrm{P} 2 \mathrm{P}$ systems need not the presence of a server for managing the network.

\subsection{JXTA entities}

The main entities of JXTA are as follows.

Peer. Any interconnected node is called peer. Peers work independently and asynchronously with other peers. Peers publish one or more interfaces that are used by other peers to establish peer-to-peer connections.

Peers can be classified in different types according to their role in the $\mathrm{P} 2 \mathrm{P}$ network as follows.

Limited Edge Peer: Peers that can send and receive messages but cannot send advertisements neither store advertisements in their cache.

Complete Edge Peer: Peers that can send and receive messages, store advertisements but cannot manage resource discovery advertisements.

Rendezvous Peer: Peers that can send and receive messages, store advertisements and moreover can manage resource discovery advertisements. Each group of peers must have at least a rendezvous peer; when a peer joins a group of peers, it automatically looks for a rendezvous in the group. Moreover, rendezvous keep a list of other rendezvous and their respective peers.

Relay Peer: This type of peers serve as intermediate nodes. Relay peer keeps routing information to other peers and sends messages to other peers that can't access directly to another peer.

PeerGroup. A peerGroup is a collection of peers that are joined to provide a secure shared environment for the participating peers. A peerGroup can decide its own policy of peer membership. Note that peers can simultaneously belong to more than one peerGroup. As in the case of peers, peerGroups offer their services such as discovery services, pipe services, peer join services, and monitoring services.

Pipes. A pipe is a virtual communication channel established between two processes. A computer connected to the network can open, at transport level, as many pipes as its operating system permits. These channels act as data links between the two communication points. Through pipes can be sent any types of objects such as strings, binary code, and java objects. JXTA offers both unidirectional, not secure pipes and bidirectional secure pipes.

Messages. Messages are objects used for communicating and interchanging data. A message is essentially an ordered sequence of tags/values, which could also include binary code (appropriately encoded).

Advertisements. The JXTA resources and services are represented using advertisements. An advertisement is a meta-data structured information (XML document), which are published with a certain lifetime that specifies its availability.

\subsection{JXTA-Overlay}

JXTA-Overlay project is an effort to use JXTA technology for building an overlay on top of JXTA offering a set of basic primitives (functionalities) that are most commonly needed in JXTA-based applications.

The proposed overlay comprises primitives for: (a) peer discovery; (b) peer's resources discovery; (c) resource allocation; (d) task submission and execution; (e) file/data sharing, discovery and transmission; (f) instant communication; (g) peer group functionalities (groups, rooms etc.); and, monitoring of peers, groups, tasks.

This set of basic functionalities is intended to be as complete as possible to satisfy the needs of JXTA-based applications. The overlay is built on top of JXTA layer and provides a set of primitives that can be used by other applications, which on their hand, will be built on top of the overlay, with complete independence. The JXTA-Overlay project has been developed using the latest updated JXTA libraries. In fact, the project offers several improvements of the original JXTA protocols/services, on the one hand, to increase the reliability of JXTA-based distributed applications [1] and to support group management and file sharing.

The architecture of the $\mathrm{P} 2 \mathrm{P}$ distributed platform we have developed using JXTA technology has these building blocks: Broker Module, Primitives Module and Client Module. Altogether these three modules form a new overlay on top of JXTA. The JXTA-Overlay system interface is shown in Fig. 2. While, the structure of JXTA-Overlay system is shown in Fig. 3.

\section{Proposed P2P e-Learning System}

\subsection{Transmission control and management in JXTA-Overlay}

The most important part in e-learning systems or virtual campus is the communication between peers (groups of students) or between teachers and students. By using the proposed JXTA-Overlay, it is possible to overcome, firewalls, routers, NATs, and bridges in the private networks. We explain in following the message transmission by the JXTA protocol.

JXTA protocol uses Universally Unique Identifier (UUID) in order to identify the peers in the private network from the Internet. The UUID is a general unique identifier. 


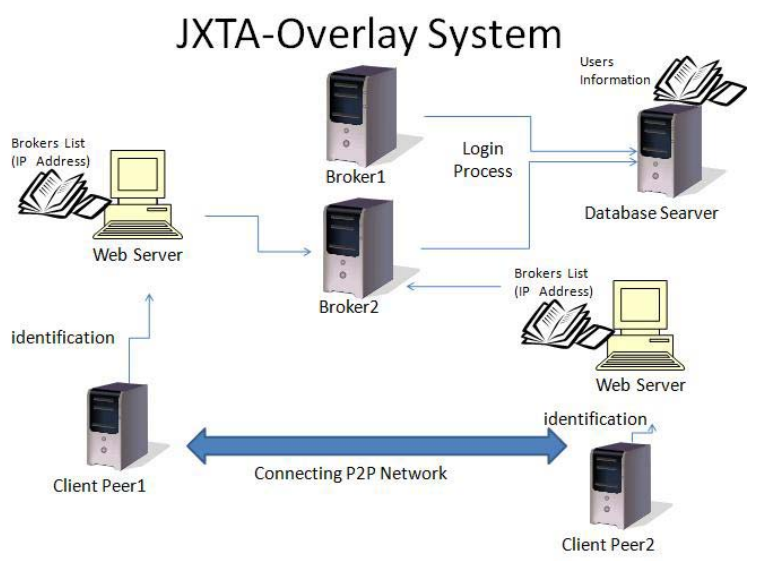

Figure 2. Structure of JXTA-Overlay system.

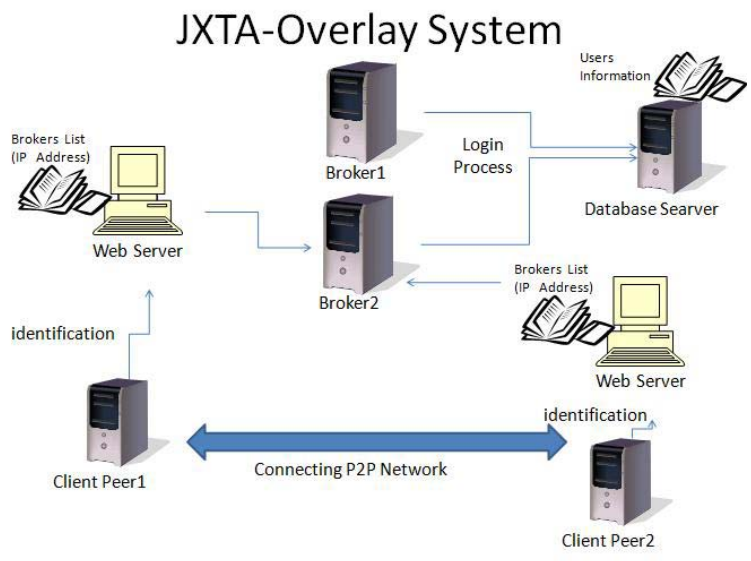

Figure 3. System image of JXTA-Overlay.

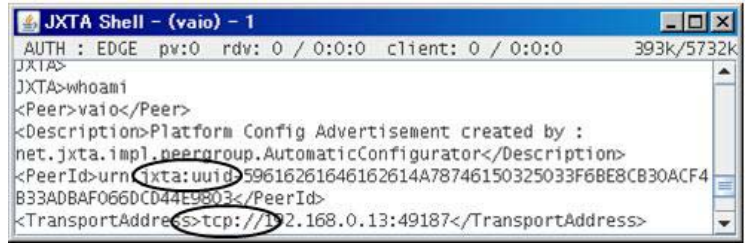

Figure 4. UUID of JXTA and TCP address.

It is generated by the NIC address of the computer, date and time. By knowing the UUID and TCP address, it is possible to make address translation. In Fig. 4 is shown the displayed UUID and TCP address by JXTA shell. The TCP/IP uses for communication socket concept. However, JXTA protocol uses the pipe method. The pipe is a communication channel for sharing the information between peers. If a peer wants to communicate with another peer first it searches for the pipe-advertisement and then the transmission and receiving of messages is done by pipe-binding.

In Fig. 5 we show the process of message sending and receiving using JXTA shell. As we will show in the following section, we implemented a control system that is able to control a peer in a private network from a peer in the Internet as showing in Fig. 6. The control targets are considered the

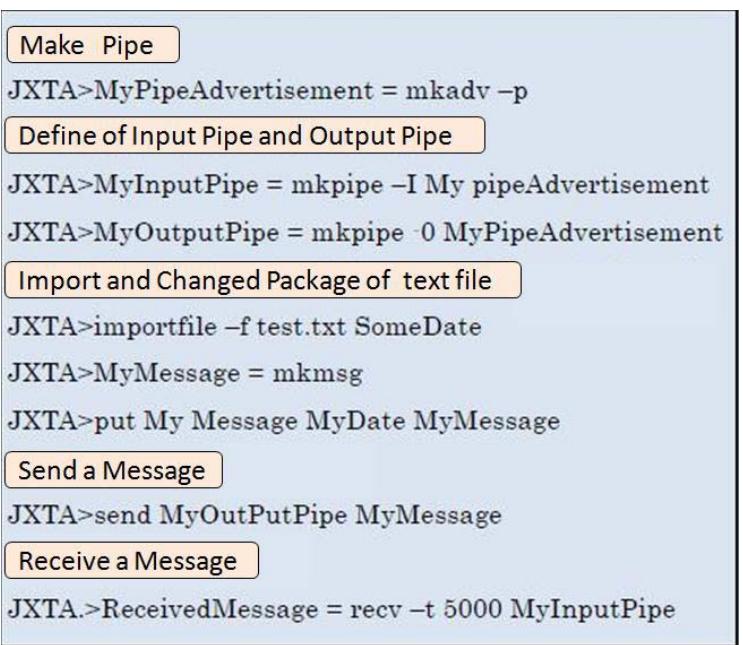

Figure 5 . Message sending and receiving by JXTA-Overlay.

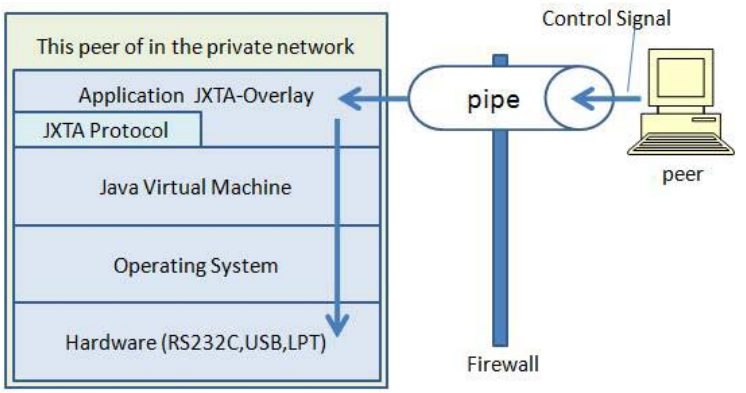

Figure 6. Implementation of remote control in JXTA-Overlay.

network devices such as RS232C port, LPT port and USB port. By implementing this kind of control system, we are able to collect data and control the peers in a wide area network. Thus, we will be able to control all devices that are connected to the peers. The application of this control function will be described in detail in the following section. We are concentrated in the control of USB devices and RS232C equipment. This is because USB devices are very popular and are used almost in every computer. Also, by using USB is possible to control the motors and LEDs. The RS232C is legal interface and many devices have implemented it.

\subsection{P2P e-learning system}

In our proposed system we combine P2P, Web and Sensor technologies. Our goal is not only to monitor and control the students' activity in a wide area network but also to stimulate and increase learners' motivation.

Our target implementation is to build a Smart Room (see 7) to control and stimulate the learners motivation. However, it will be a little bit expensive for our project. For this reason, we proposed and implemented a SmartBox environment as shown in Fig. 8. The SmartBox uses RFID 


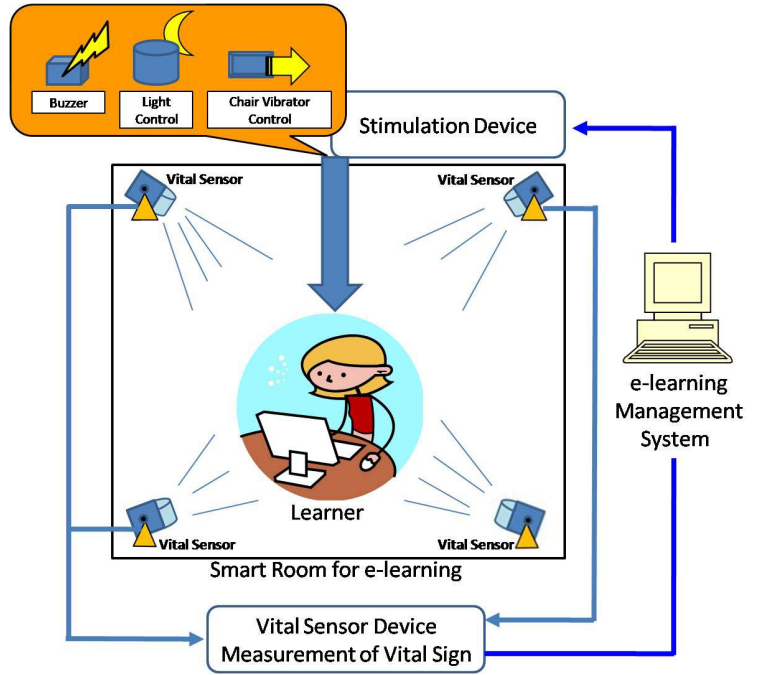

Figure 7. Smart room.

(Radio Frequency Identification) and Vital Sensors. In our system, we use JXTA-Overlay for communication between peers and sending the control signal. We have implemented the following function to control and improves the learners motivation

- Vibration Function: this function is achieved by using the vibration function of PDA and Cellular Phone or by moving the learner's chair. This is done when the learner does not use the computer keyboard for a determined period of time. In this case, it is considered that the learner is sleeping or loosing his concentration.

- Smell Function: by this function, the system change the mood of the learner.

- Sound Function: using this function, a relaxing sound is emitted from the speaker to create a relaxing environment.

- Room Light Control: by changing the brightness or lighting of the room the student's mood and the concentration is controlled.

- Temperature Control: this function controls the temperature of the room. This is very important function, because the mood of the student is related with the room condition.

The learner's situation is quantified and measured by the vital sensor and the e-learning management computer checks the learner's motivation level. Then the proposed elearning control system uses physical stimulation to change the mood of the learners.

The learner identification is carried out by RFID. Using the RFID, the system can check whether the learner is in the room or not. In the case when the learner doesn't return to the room for a long time, the system automatically sends an e-mail to the cellular phone of the learner and urges him/her to return to the study room.

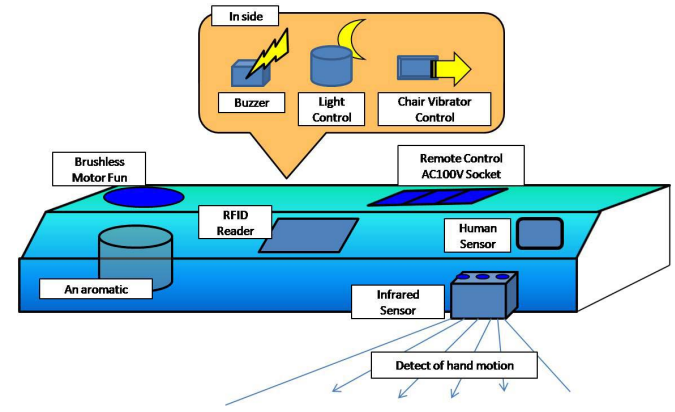

Figure 8. SmartBox functions.

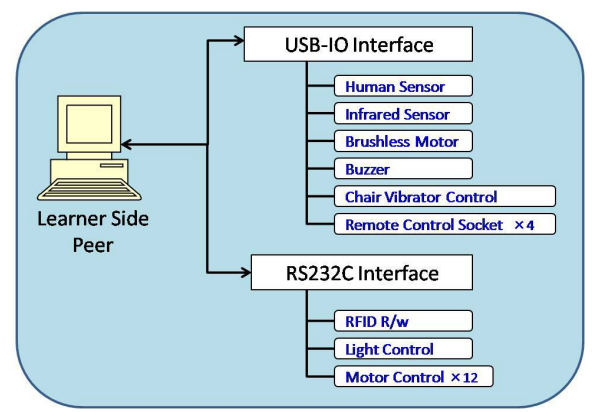

Figure 9. Functions and interfaces of SmartBox.

\subsection{SmartBox design, implementation and inte- gration with $\mathbf{P 2 P}$ system}

The size of SmartBox is $50 \times 10 \times 15 \mathrm{~cm}$ and is put in the learner's desk. SmartBox is equipped with different sensors for sensing learner situation and device that are used for learner's stimulation.

The SmartBox shown in Fig. 9 has the following sensors and functions.

- Human Sensor: for detecting whether the learner is in the room or not.

- Infrared Sensor: for detecting the learner's hand.

- RFID Sensor: for identifying learner's IC tag card.

- Chair Vibrator Control: for vibrating the learner's chair.

- Light Control: for adjusting the room light.

- Aromatic Control: this function is used for controlling the room smell.

- Buzzer Control: to emit relaxing sounds.

- Remote Control Socket: for controlling AC 100V socket (on-off control).

A snapshot of the implemented SmartBox is shown in Fig. 10. The SmarBox can detect the learners movement by using human sensor and infrared sensor. The human sensor are used for controlling the body movement of the learner. On the other hand, the infrared sensor controls the hand motion of the learner. The RFID sensor can read IC tag information and record the learner's study history. 


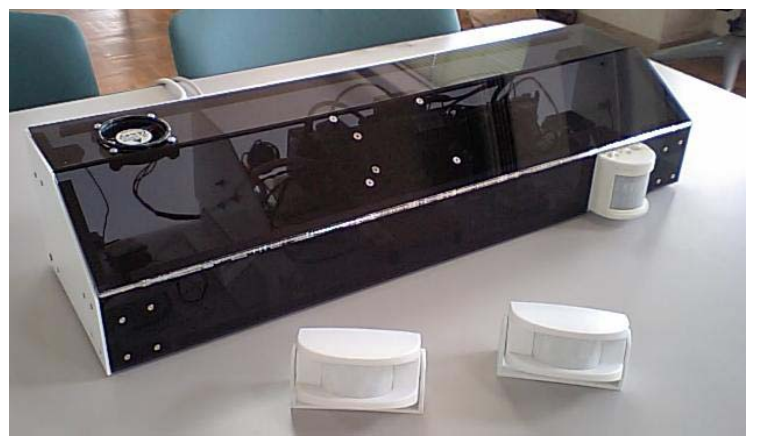

Figure 10. A snapshot of implemented SmartBox.

\section{Conclusions and Future Work}

In this work, we presented the design and implementation of JXTA Overlay P2P e-Learning system. The proposed system is able to overcome Firewalls, Routers, Bridges, NATs by using JXTA technology and can control and stimulate the learners motivation by using proposed and implemented SmartBox. By using the RFID, it is possible to read and write the IC tag card and manage the learner's study history. SmartBox is integrated with the P2P system by benefiting features of $\mathrm{P} 2 \mathrm{P}$ systems such as direct communication among peers.

In the future, we want to add other effective stimulation for each learner in the IC tag card. Also, the ServoMotor has 12 control ports, so we will use some of its ports for implementing other stimulating functions.

We plan to evaluate the proposed system in a real environment of virtual campuses. We also would like to evaluate the proposed system for different scenarios where monitoring and control of students in the wide area is important.

\section{References}

[1] F. Xhafa, R. Fernandez, T. Daradoumis, L. Barolli, S. Caballe, "Improvement of JXTA Protocols for Supporting Reliable Distributed Applications in P2P Systems", Proc. of NBiS-2007 (Regensburg, Germany), LNCS 4658, pp.345-354, September 2007.

[2] J. Ma, L. Barolli, M. Shizuka, and R. Huang, "A Pure P2P Synchronous Collaborative System", Journal of Applied System Studies (JASS), Vol. 5, No. 2, pp. 133145, July 2004.

[3] IBM Corporation, "Making P2P Interoperable: Creating JXTA Systems Design P2P Systems that Extend Beyond Traditional Network Boundaries", 2007.

[4] Sun Microsystems, "JXTA Java Standard Edition v2.5: Programmers Guide", June 2007.

[5] H. Bal, H. Casanova, J. Dongarra, and S. Matsuoka, "Application-Level Tools", In Foster et al., eds, The Grid: Blueprint for a New Computing Infrastructure, Chapter 24, pp. 463-489, Morgan Kaufmann, 2003.
[6] S. M. Lui and S.H. Kwok, "Interoperability of Peer-toPeer File Sharing Protocols", SIGecom Exch.Journal, Vol. 3, No. 3, pp. 25-33, ACM Press, New York, NY, USA, 2002.

[7] F. Le Fessant, S. Handurukande, A. M. Kermarrec, L. Massouli, "Clustering in Peer-to-Peer File Sharing Workloads", Peer-to-Peer Systems III, Lecture Notes in Computer Science, Vol. 3279/2005, pp. 217-226, 2005.

[8] K. P. Gummadi, R. J. Dunn, S. Saroiu, S. D. Gribble, H. M. Levy, J. Zahorjan, "Measurement, Modeling, and Analysis of a Peer-to-Peer File-sharing Workload", Proc. of 19-th ACM Symposium on Operating Systems Principles, pp. 314-329, ACM Press, New York, NY, USA, 2003.

[9] T. Karagiannis, A. Broido, M. Faloutsos and K. Claffy, "Transport Layer Identification of P2P Traffic", Proc. of the 4-th ACM SIGCOMM Conference on Internet Measurement, pp. 121-134, Taormina, Italy, ACM Press, 2004.

[10] CALAT Project, Nippon Telegraph and Telephone Corporation, http://www.calat.com/.

[11] CALsurf, NTT Software Corporation, http://webbase. ntts.co.jp/.

[12] The University of The Air, http://www. u-air.ac.jp/.

[13] California Virtual University, http://www.california. edu/.

[14] WIDE University, School of Internet, http://www. sfc.wide.ad.jp/soi/contents.html.

[15] Blacboard, http://www.blackboard.com/webct.

[16] L. Barolli, A. Koyama, "A Web-based Distance Learning System Using Cooperative Agents", Chapter in Encyclopedia of Online Learning and Technology, Idea Group Inc. Publishing, Vol.4, pp.430-439, April 2005.

[17] L. Barolli, A. Koyama, A. Durresi, G. De Marco, "A Web-based E-learning System for Increasing Study Efficiency by Stimulating Learner Motivation", Journal of Information Systems Frontiers, Springer Publishers, Vol. 8, No. 4, pp. 297-306, September 2006.

[18] D. Brookshier, D. Govoni, N. Krishnan, J.C Soto, "JXTA: Java P2P Programming", Sams Publishing, 2002. 